Research Article

\title{
Intelligent Perception System of Big Data Decision in Cross- Border e-Commerce Based on Data Fusion
}

\author{
Xiaheng Zhang, ${ }^{1,2}$ Dongpeng Xu $\mathbb{D}^{3}$ and Lin Xiao ${ }^{2}$ \\ ${ }^{1}$ College of Business and Trade, Nanchang Institute of Science and Technology, Nanchang, 330108 Jiangxi, China \\ ${ }^{2}$ Management School, Northwest University of Political Science and Law, Xi'an, 710122 Shaanxi, China \\ ${ }^{3}$ Faculty of Finance, City University of Macau, Macao, 999078 Macao, China
}

Correspondence should be addressed to Dongpeng Xu; f20092100138@cityu.mo

Received 29 April 2021; Revised 28 May 2021; Accepted 12 June 2021; Published 25 June 2021

Academic Editor: Mu Zhou

Copyright ( 2021 Xiaheng Zhang et al. This is an open access article distributed under the Creative Commons Attribution License, which permits unrestricted use, distribution, and reproduction in any medium, provided the original work is properly cited.

There is a gap between supply and demand in cross-border e-trading platforms. The so-called supply and demand gap refers to the existing good companies that cannot meet the needs of buyers. Cross-border e-commerce, the buyer's purchase demand is often included in the buyer's behavior, such as searching for goods by pressing buttons, clicking price, category and other means, and on-site delivery time. It is important for CBEC to analyze the buyer's needs to protect the procurement and provide the seller with reference to supply, so as to solve the supply and demand gap between the buyer and the seller. This paper mainly studies the CBEC big data decision intelligent perception system based on data fusion. It is the same as the innovation process of better application of data fusion to the e-commerce business model. At the same time, the key technologies of data extraction, conversion, data warehouse, and front-end display in the system construction are analyzed and designed. This paper mainly uses data fusion algorithm, data fusion network model, quaternion method, big data decision intelligent sensing system framework design experiment, and CBEC user experiment to study the CBEC big data decision intelligent sensing system based on data fusion, and also with the development of CBEC, I hope more people can participate in CBEC practice. The results show that with the increase of data volume of the CBEC platform from 2016 to 2020, the construction of the CBEC platform business of big data production intelligent perception system considers the situation of users from seven aspects. With the increase of consumption, domestic consumers are increasingly pursuing a better life, cross-border import e-commerce ushered in a great development era.

\section{Introduction}

Cross-border e-commerce is an important part of Internet services. With the development of the Internet, CBEC has been extended to all aspects of the economy and society, as well as its impact on the manufacturing industry, and other industries are becoming more and more important. Internet users have become a common behavior to shop through the CBEC platform. The basic function of the CBEC platform is to provide a platform for the buyer and seller to trade on the Internet. The criteria to evaluate whether the CBEC platform can provide users with good service include user satisfaction and user experience after-sales service. One of the most important aspects is that the seller users can provide the goods that the buyer users want to buy. Users usually use the search engine of the CBEC platform to search for the items they want to buy and then select the items that meet the users' wishes to purchase in the search results. If users cannot search for the goods they want to buy on the platform, they will give up their willingness to purchase this time or switch to other CBEC platforms for purchase. In order to solve this practical problem, it is necessary to analyze the supply and demand of all goods on the platform on the CBEC platform and solve the problem of asymmetric information between buyer and seller users through supply and demand analysis.

In the current situation of explosive growth of Internet data, it is required that the Internet industry store and process data efficiently. Every day, the CBEC platform will produce a large number of data, such as user behavior data, 
transaction data, updated goods information, user information, and customer service information. When these data are stored, there is a need for an efficient platform for data extraction and analysis. According to statistics, the data volume in 2014 will reach $4 \mathrm{zb}$, and $34 \%$ of the general data of the Internet can be understood and applied, which has a high utilization value. These data are obtained through CBEC, social networks, video, and entertainment. However, only about $7 \%$ of these valuable data are effectively used, and such information has high analytical value. Of all data, only 1 percent of the data is analyzed.

With the development of big data and cloud computing, the consumption of data storage resources and computing cost has been greatly reduced. The construction of these databases has been improved, and data storage is not the main problem in the era of big data. When data acquisition is more and more, how to use data effectively becomes one of the most important problems. Liao et al. believe the results of the 2014 data fusion competition organized by the IEEE Society for image analysis and data fusion technology committee. As in previous years, the technical committee of the space debris federation organized a data fusion contest aimed at fostering new ideas and solutions for multisource remote sensing research. The founders considered the fusion of multiresolution and multisensor between optical data with $20 \mathrm{~cm}$ resolution and long-wave infrared hyperspectral data of 1meter resolution. The competition was proposed as a twotrack race: one goal was accurate land cover classification, and the other was to seek innovation in the fusion of thermal hyperspectral and color data, but lack of specific data [1]. Semmens et al. believe that the fusion method of big data sensor estimates the evapotranspiration of vineyards. Combining with Landsat 8, MODIS, goes data, the estimated value of ET of field size is provided. The simulated surface energy flux is in good agreement with the ground flux measurement value, and the spatial distribution of ET is consistent with the output estimation. Therefore, the early inference may be due to the coverage of crops between vines, but the necessary experimental data is not available [2]. Bareinboim and Pearl believe that the concept, principles, and tools of current causal analysis methods are unified, and the new challenges brought by big data are concerned. In particular, the data fusion and merging of the problem data we solve are collected in different conditions to obtain effective answers but lack of numerical analysis content [3]. Berkow et al. think that the performance measurement of the trunk line is the key problem of traffic system management, traveler information, and real-time situation-aware routing. In many urban areas, the latest information on the state of the motorway is available, taking into account the large number of trips that have taken place on these facilities. Since nearly 40 percent of the mileage of vehicles in the United States occurs on the main road, similar information is required, which is not only available for travelers but also available for traffic engineers and managers. Since many main roads are equipped with drive traffic lights, it has been explored to use installed sensors as a source of traffic volume, occupancy, or speed data to inform the main road performance system. With this in mind, it is possible to take advantage of the availability of mobile detector geolocation data, which include automated vehicle positioning systems for buses or taxi fleets, or mobile phones or other GPS-type equipment. Demonstrate the potential value of fusing fixed and mobile monitoring system data, but some of the discussions are not accurate [4].

The innovation of this paper is to study the intelligent perception system of CBEC big data decision-making based on data fusion, such as data fusion algorithm, data fusion network model, quaternion method, and big data decision intelligent perception system design experiment and CBEC user experiment, and at the same time, it is aimed at encouraging more entrepreneurs to join CBEC. The research on the decision-making of the CBEC model can help cross-border enterprises choose the right business model, reduce the possibility of decision-making mistakes, and improve the success rate of CBEC business [5].

\section{Data Fusion Algorithm}

2.1. Data Fusion. Data fusion in business big data is a process of getting more accurate description of the fusion information of perceived objects through a certain rule, intelligent analysis of multilevel optimization, and finally, the user's needs are completed [6]. In business big data, the perceived information obtained by data fusion technology is usually more persuasive than the data collected and analyzed by a node. Data fusion can ensure the accuracy of perceived data, reduce the network data traffic and reduce redundant data in the network, and play an important role in making reasonable decision-making for application [7, 8]. In the big business data, the energy of nodes is limited. Multidimensional data collected from different nodes can be aggregated by using data fusion technology, so as to eliminate redundant data and reduce the data communication volume in the network to reduce the energy loss [9]. Data fusion technology can be combined with a variety of technologies: data fusion is often combined with clustering technology of topology. When cluster head collects data collected in cluster, it will be fused, and the fusion results will be transmitted to base station. Decision big data fusion by base station can be shown in Figure 1.

\subsection{Data Fusion Network Model}

2.2.1. Communication Energy Consumption Model. KPCA can change some nonlinear data into linear data by dimension increasing, which solves the problem that PCA cannot effectively extract principal components when dealing with some nonlinear problems [10]. Meanwhile, due to the nonlinear transformation, KPCA may extract more principal components than PCA, which can be expressed as the loss of energy $E$ when a node transmits $n$ bit data to a node whose distance is

$$
E(n, a)=E \times n+\varepsilon \times n \times a^{2},
$$

where $e$ is the unit of transmission (sending or receiving) and the energy lost by the node when bit data is transmitted; $M$ is the power amplification factor of the free space transmission 


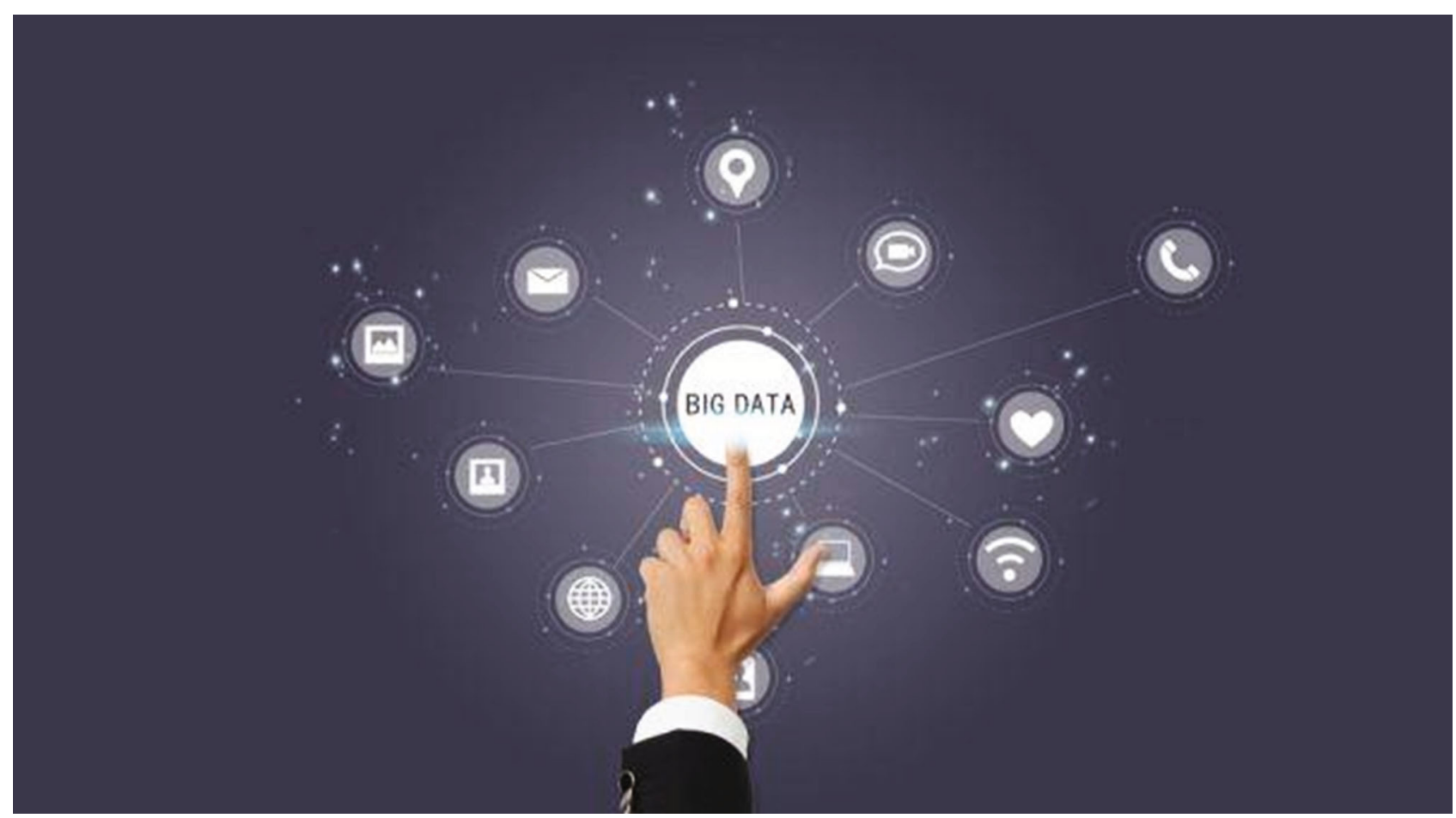

FIgURE 1: Internet edge computing (http://alturl.com/qfxtf).

model; $M$ is the power amplifier factor of the multipath attenuation model; $a$ is the threshold, when $a<A$; the spatial transmission model has been adopted; otherwise, the multidimensional suppression transmission model should be adopted $[11,12]$. On the contrary, the calculation formula for the energy loss of $a$ bit data received by the receiving node is as follows:

$$
H=a E
$$

In addition, the calculation of threshold satisfies the formula:

$$
A=\sqrt{\frac{m}{M}}
$$

2.2.2. Optimal Number of Cluster Heads. Suppose that there are $m$ randomly deployed sensor nodes in the $H=a * a$ monitoring area, $a$ is the number of cluster head nodes in the network, that is, the number of clusters, and the energy consumed by data fusion is $f$ [13]. Then, the energy consumption of each round of cluster head in the network is expressed as

$$
F=n \times E\left(\frac{M}{a}-1\right)+n \times \frac{M}{a}+n \times \varepsilon b^{2},
$$

where $B$ is the average distance from the cluster head to the base station, and $B$ is calculated as follows:

$$
b=\iint_{s} \sqrt{x^{2}+y^{2}} \frac{1}{s} d x d y .
$$

For the completion time of the task, it is not only related to the upload completion time of edge calculation but also related to whether the previous upload edge calculation is completed [14].

2.2.3. The Design of Ceoda Algorithm. According to the ratio of the residual energy to the average residual energy in each region, the nodes are divided into candidate cluster head nodes and common nodes. Candidate cluster head nodes participate in cluster head election. Using the idea of fuzzy logic, the input variables are the average residual energy of the node, the distance from the node to the receiver, and the number of neighbors around the node, the opportunity value of all candidate cluster head nodes is calculated in this round, and the more appropriate cluster head node is selected [15]. The energy consumption $E$ and the whole network of a complete cluster in each round can be calculated; the energy consumption of each round $W$ is expressed as

$$
E=W+\left(\frac{M}{a}-1\right) E
$$

Therefore, by minimizing $E$ and making its derivative to a 0 , the optimal number of cluster heads $n$ is calculated, which is expressed as follows:

$$
n=\sqrt{\frac{M}{2 \pi}} \frac{A}{b}
$$

In the range of $N$, the more neighbor nodes $a$, the more extensive the information collected and the wider the information coverage, so it is more suitable to be elected as cluster 


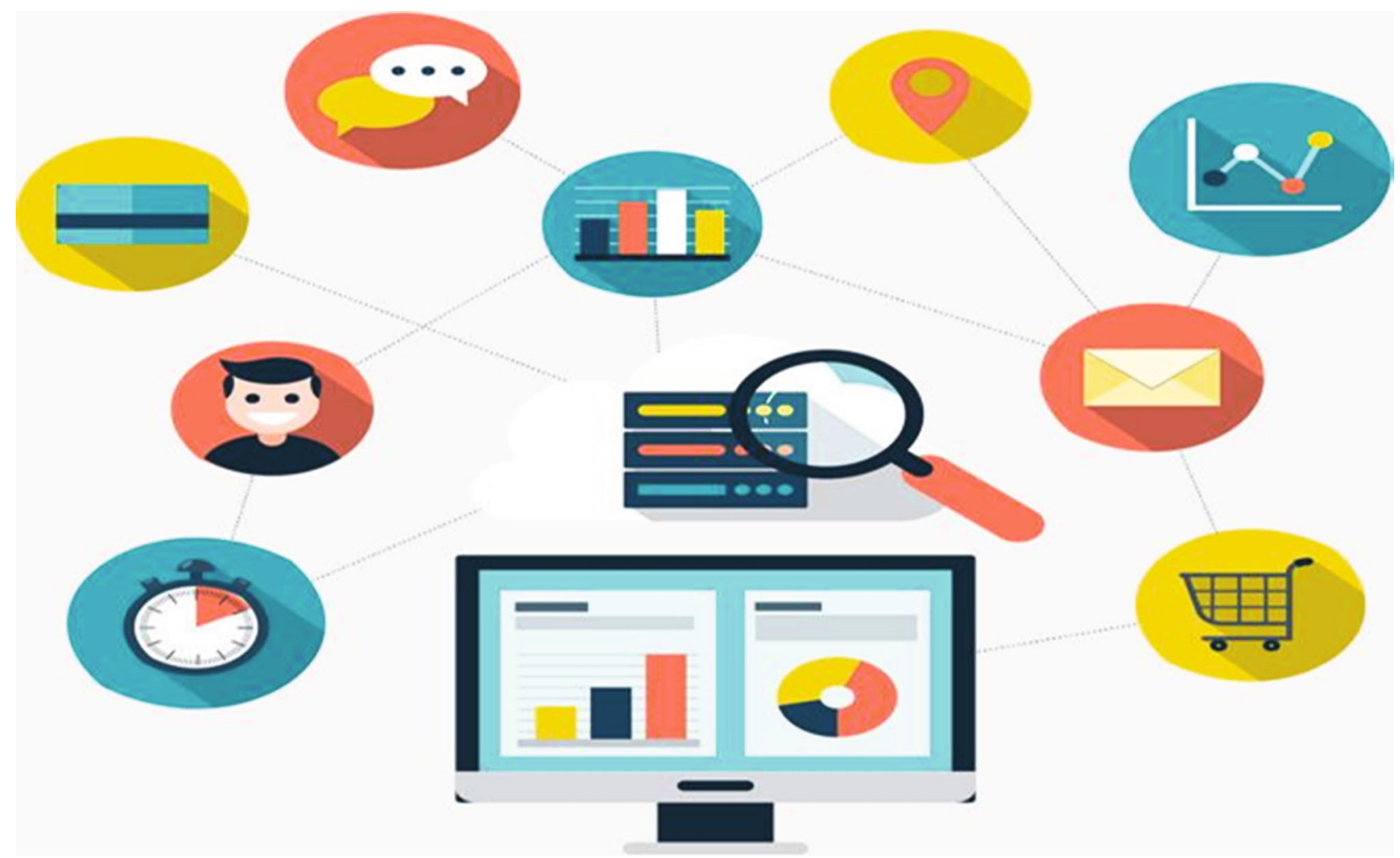

FIgURE 2: Internet business big data (http://alturl.com/xtpfp).

head node. The minimum radius of $N$ ideal cluster is calculated as follows:

$$
N=\sqrt{\frac{A}{\pi \times a}} .
$$

When sampling data arrives at a specific time slot, an adjustable data similarity ratio $C$ is set at $F$. The selection strategy is as follows:

$$
f \leq h+h \times(1-c) .
$$

$F$ is the actual monitoring data of a node in a cluster in the current time. $H$ and $C$ represent the maximum and minimum monitoring values of adjacent common nodes, respectively [16].

2.3. Big Data. Big data, as a hot topic in academic research, has attracted the full attention of experts and scholars around the world, and the relevant analysis and research literature have increased year after year $[17,18]$. Foreign countries have made in-depth research on the following three aspects, including the basic theory of big data. It usually focuses on the origin and development, basic definition, main characteristics, and basic structure of big data. The characteristics of big data are as follows: large volume, many kinds, and poor accuracy; the reason why big data has attracted more and more attention is that it has great social influence and significance in terms of cultural tradition, professional technology, academic status, and data and information research and analysis; the traditional data information collection, data information acquisition, data information research and analysis, and data information application technology cannot meet the demand [19]. Analysis and research on the quality of big data, there are a lot of low-quality data information in large-scale data databases and the Internet. Big data is shown in Figure 2.

For big data problems, we can use the data preprocessing to deal with and solve the problem of big data quality. The experimental model of big data quality obtained by a kind of operation process can be used to ensure the selection and adaptation of data quality, the evaluation of comprehensive quality of big data, and the analysis and research of reference standards [20].

2.4. Data Fusion Quaternion Method. The quaternion theory is to transform the dynamic measurement problem which is inconvenient to measure into the change mode of the object rotating around the fixed point easily. According to the above definition of quaternion, the quaternion $G$ can be converted into the following form:

$$
G=g_{0}+g_{1} i+g_{2} j+g_{3} k
$$

Its fixed-point rotation can be described as the rotation of a coordinate or a vector relative to a certain coordinate system:

$$
G=\sin i \cos a+\sin j \cos \beta .
$$


TABLE 1: The field meaning of filtering extraction.

\begin{tabular}{lccc}
\hline Protocol type & Key field & Field meaning & Purpose \\
\hline Radius & 0x0608 & Broadband account & Identify user \\
Http & Network layer & User's public IP & Matches broadband \\
& Host & Host domain name & User identity \\
& User agent & User description & Terminal \\
\hline
\end{tabular}

TABLE 2: User identity key steps.

\begin{tabular}{lc}
\hline Serial number & Step \\
\hline 1 & DPI data cleaning \\
2 & Protocol analysis \\
3 & Message suffix filtering \\
4 & Broadband account extraction of radius message \\
5 & Unified decoding \\
6 & Basic message information \\
\hline
\end{tabular}

Indicates the direction of rotation of the instantaneous shaft, therefore, quaternion can not only represent the direction of rotation of the rotation axis but also represent the size of the rotation angle. Now, this rotation relationship is expressed by the following operation methods:

$$
H^{a}=G H^{n} G^{a} .
$$

In order to make the filtering algorithm suitable for the nonlinear system, it is necessary to improve it to a certain extent. The Kalman filter has the function of simple algorithm and easy implementation, so either the classical Kalman filter algorithm or the improved extended and unscented Kalman filter algorithm will be applied in various fields, such as navigation guidance, target tracking, and integrated navigation. Let the state equation and measurement equation of the stochastic discrete system at $k$ time be

$$
H_{k}=F_{k} X_{k}+W_{k}
$$

The general solution process of the Kalman filter algorithm is as follows:

$$
Y_{k, k-1}=\varphi_{k, k-1} Y_{k, k-1}
$$

State estimation equation:

$$
Y_{k, k-1}=Y_{k, k-1}+K_{k}\left(F_{k}-W_{k} Y_{k, k-1}\right)
$$

\section{Related Experiments of CBEC Big Data Decision-Making Intelligent Perception System}

3.1. Cross-Border e-Commerce. Cross-border e-commerce is derived from CBEC, personal purchase, and other modes. With the development mode of different conditions becom- ing more and more mature, China's CBEC market is also gradually evolving, which makes researchers explore it from the development process, development ideas, and other aspects. The problems and countermeasures in the development process show that China's e-commerce has broad prospects and great potential, but the development of crossborder logistics is slow. It has seriously restricted the further development of cross-border trade and new logistics, while foreign warehouses are emerging. With the development of the times, it can solve many problems that traditional cross-border logistics cannot solve, such as time-consuming, high cost, and heavy custom clearance. Cross-border ecommerce enterprises also face some problems in using foreign warehouses. For example, the wrong choice of foreign storage facilities and the operation mode of CBEC companies have brought many obstacles to the future operation.

3.2. Cross-Border e-Commerce User Identity Keywords. For a large-scale data analysis system, because there is a lot of unnecessary information in the local broadband business data, in order to meet certain strategic needs, the required data must be deleted for effective data analysis to ensure the accuracy and efficiency of extraction. Most broadband users' network traffic is DPI, which is used to analyze data packets and extract relevant information, evaluate the type of packet protocol, and filter and delete packets other than HTTP and nonbeam, such as FTP, DNS SMTPitp and Nast. Then, other HTTP packets are analyzed through HTTP application layer messages, and fields such as URI, host, UA, refer, cookie, and content are downloaded. The meaning of the extracted fields is shown in Table 1.

For example, in the URI field, ICT, GIF, PNG, JS, JPEG, CSS, and other formats are used as extensions to require image resources and website styles. Since the traffic data are all requests of users for unified data resources, they do not contain enough information about the user's identity, so it is useless to extract the user's identity information, as shown in Table 2.

3.3. Big Data Decision Intelligent Perception System Framework. On the basis of the unique advantages of having more complete user online shopping traffic than other CBEC platforms, operators provide broadband users with personal online shopping recommendation, which not only provides reliable traffic promotion methods for operators but also provides high-quality data services for home broadband users, which has far-reaching significance and value in the field of CBEC [21, 22]. Through the analysis and mining of home broadband user traffic, and through a series of operations such as user feature extraction based on deep packet analysis, 


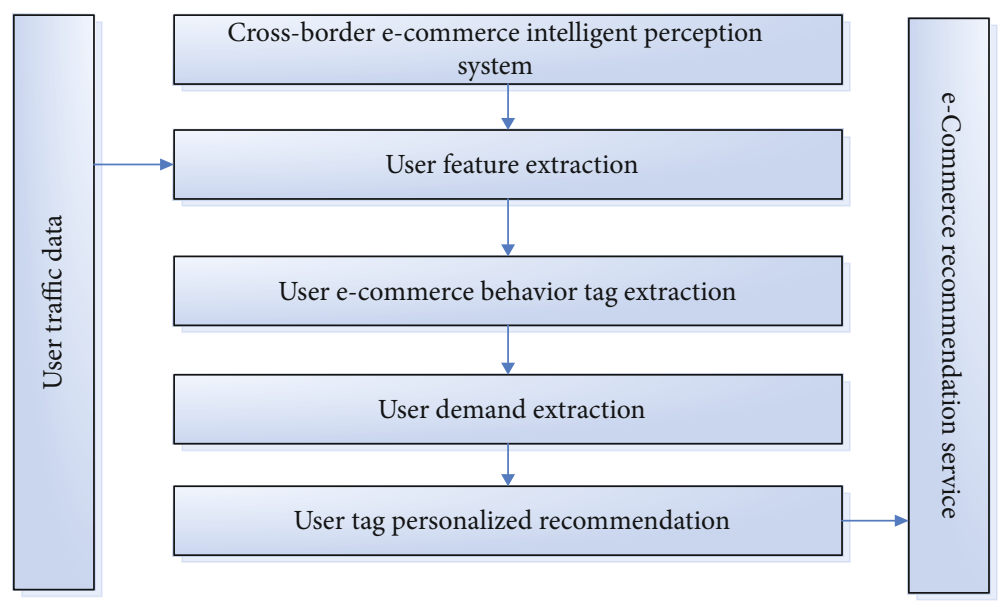

Figure 3: Platform user management module.

TABLE 3: Ranking of cross-border e-commerce platforms in China.

\begin{tabular}{lc}
\hline Popularity & e-Commerce platform \\
\hline 1 & Tmall global \\
2 & NetEase koala \\
3 & JD global \\
4 & Little red book \\
5 & Ocean terminal \\
6 & Vipin international \\
\hline
\end{tabular}

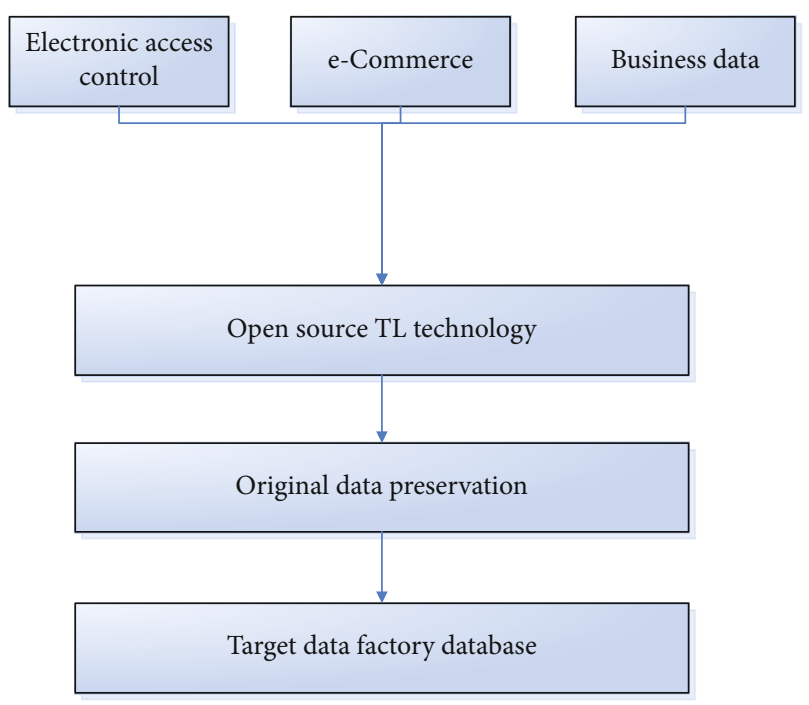

Figure 4: Platform user login.

tag extraction based on user e-commerce behavior, and personalized recommendation based on user tag, the personalized e-commerce recommendation service for home broadband users based on big data analysis is finally realized. The basic framework flow is shown in Figure 3.

3.4. Evolution of Cross-Border Import e-Commerce. The development of CBEC in China has experienced five stages: germination, birth, development, standardization, and standardization. e-Commerce begins with the purchase of foreign goods for Chinese foreign students. They went to foreign supermarkets to buy foreign goods and sent them to China by meat express. With the emergence of national food security and the increase of consumption, the demand of Chinese population for foreign goods increases, and CBEC begins to rise. The state has issued a series of important policies to adapt to the development of CBEC, opening up crossborder registration licenses successively, launching CBEC market, and large amount of funds flowing into crossborder areas. China's CBEC import has entered the peak of growth, and various CBEC companies have emerged. The state then issued a series of policies to regulate the behavior of CBEC enterprises. A number of small- and mediumsized CBEC enterprises began to close, and CBEC entered the brand washing and ranking period, users gradually gather to a complex platform and vertical classification of CBEC enterprises import, and the industrial chain optimization has entered a new stage.

\section{Intelligent Perception System of Big Data Decision in CBEC}

4.1. Cross-Border e-Commerce Trading Enterprises in China. According to the survey of popularity ranking of China international e-commerce platform in 2019, Tmall international reputation ranks first, NetEase koala ranks second, and JD ranks third in the world, while Hong Kong and JD, respectively, get the fourth and fifth popularity. As a benchmark enterprise of China's e-commerce, Tmall and JD gather a large number of users. Their cross-border import business will grow faster than other companies. However, as a comprehensive procurement platform, they are limited in meeting the personalized needs of cross-border users. Haigou koala relies on NetEase to select CBEC users from NetEase user base. NetEase koala takes the way of direct and independent procurement in commodity supply, which makes the goods have excessive production cost and can transform users. Tmall international, JD global, and NetEase koala are the leading enterprises of CBEC in China. Xiaohongshu 
TABLE 4: e-Commerce platform user rate questionnaire.

\begin{tabular}{ccc}
\hline Year & Number of users (must) & User rate \\
\hline 2016 & 40 & $7.34 \%$ \\
2017 & 65 & $12.45 \%$ \\
2018 & 76 & $13.25 \%$ \\
2019 & 78 & $15.66 \%$ \\
2020 & 80 & $18.88 \%$ \\
\hline
\end{tabular}

TABLE 5: Weighting table of influencing factors.

\begin{tabular}{lc}
\hline Factors & Weight \\
\hline Users & 8 \\
Products & 7 \\
Experience & 5 \\
Talents & 6 \\
Technology & 7 \\
Capital & 5 \\
Logistics & 6 \\
\hline
\end{tabular}

works with foreign end-end e-commerce to make vertical segmentation and finally obtain market recognition according to user needs. Vertical e-commerce imports represented by Xiong Hongtu, foreign terminals, and darling constitute the basis of CBEC import in China. The popularity ranking of e-commerce platform is shown in Table 3.

As a cross-border platform and e-commerce, ecommerce can continue to promote economic globalization and play an important role in promoting the development of foreign trade. In terms of development level, the development level of CBEC in developed countries in Europe and America is significantly higher than that in China, However, with the information fusion technology, the decision intelligent perception system is a high-level application based on the existing informatization of the scenic spot. It has strong practicability and advanced nature and can meet the requirements of cross-border business decision support, business data analysis, traffic prediction, data management, and system management. Therefore, the intelligent decision system of scenic spot decision-making based on business intelligence is designed. The overall framework of the system is shown in Figure 4.

The data required by the prediction submodule is distributed in business systems such as electronic access control, ecommerce, and business data or public platforms. In order to make accurate prediction of CBEC by using these data, the ETL Technology must be used to extract and transform these data and then load them into the data warehouse. In order to meet the actual information data decision-making status of CBEC, the ETL Technology must be used to extract and transform these data, so each business system is designed to use different database software storage, which has common representativeness.

4.2. Number of Cross-Border e-Commerce Internet Users. China has a huge Internet user base, so China's CBEC import has a huge consumer market. Since 2016, the number of users of the e-commerce platform has increased significantly. This is due to the vigorous promotion of crossborder enterprise development by China's e-commerce and the high attention paid by various departments to the development of CBEC. The situation of users of the CBEC platform is shown in Table 4.

From Table 4, we can see that the number of Internet users in China reached 610 million in 2016, among which CBEC users exceeded 40 million, and the penetration rate of CBEC users was 7.34\%; in 2017, the number of Internet users in China reached 640 million, of which the number of CBEC users reached 65 million, and the penetration rate of CBEC users reached 12.45\%; in 2018, the number of CBEC users in China increased to 76 million, with the penetration rate of CBEC users reaching 13.25\%; in 2019, the number of CBEC users in China increased to 780 million, and the penetration rate of CBEC users reached $15.66 \%$; in 2020, the number of CBEC users in China will increase to 80 million, and its permeability will be increased to $18.88 \%$.

4.3. Construction of Cross-Border e-Commerce Decision Intelligent Perception System Model. The construction of the CBEC decision model is the application of cross-border import decision matrix in the e-commerce decision model, and it is a practice of the optimal scheme selection. The expert method is used to summarize all the alternative business models. The evaluation criteria are replaced by common factors affecting e-commerce enterprises' decision-making, namely, flow introduction, supply chain integration ability, core management personnel industry experience, Internet product development ability; capital, logistics, and talent factors play an important role in the selection of the CBEC model as shown in Table 5.

It can be seen from Table 5 that the expert method is used to assign values to each factor, and weights are given from 1 to 10 according to the importance of the influencing factors. The weights of each influencing factor are calculated by the average value after the expert group members assign values to them, respectively. China's cross-border import and ecommerce mainly include "online tariff import" and "direct import." Therefore, China's cross-border import and export trade mainly includes "online import" and "direct import" bonded custom clearance and direct mail custom clearance. Therefore, an intelligent system for CBEC big data decisionmaking is established. The flow chart is shown in Figure 5.

The construction of the cross-border decision-making model of import e-commerce is the application of the cross-border business model decision-making matrix in ecommerce, and it is the practice of system optimization selection: traffic (users), supply (product organization supply), experience (industry experience of core executives), talent, technology (Internet product development ability), capital, and logistics. The expert method is used to give weight to the seven elements. The influencing factors are evaluated according to the evaluation criteria, that is, the influencing factors are evaluated according to the actual situation of the enterprise to be diagnosed. The highest score is the theoretical optimal option. The "influencing factors" and evaluation criteria are determined as shown in Figure 6. 


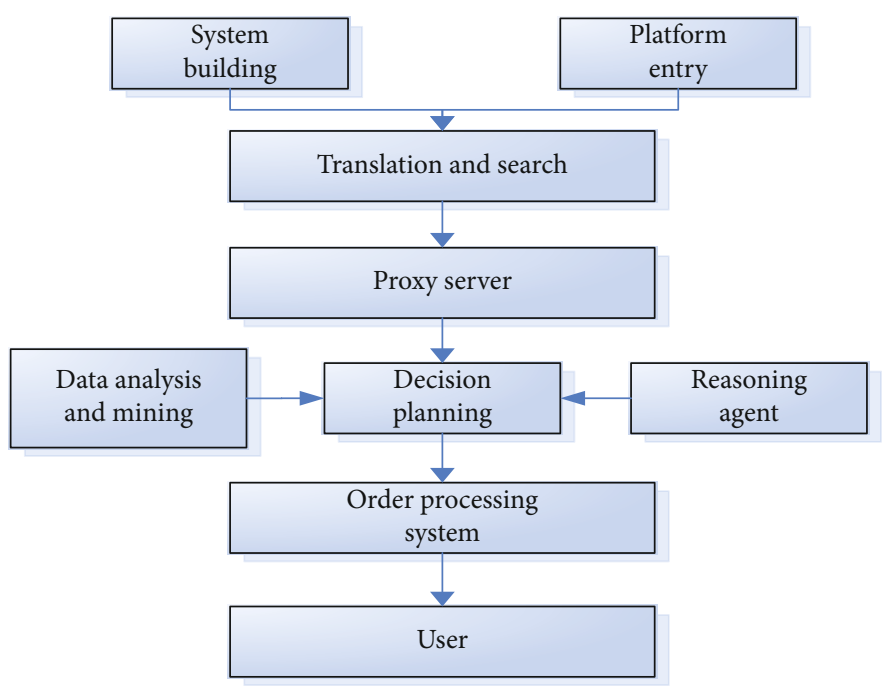

Figure 5: Platform collaborative management.

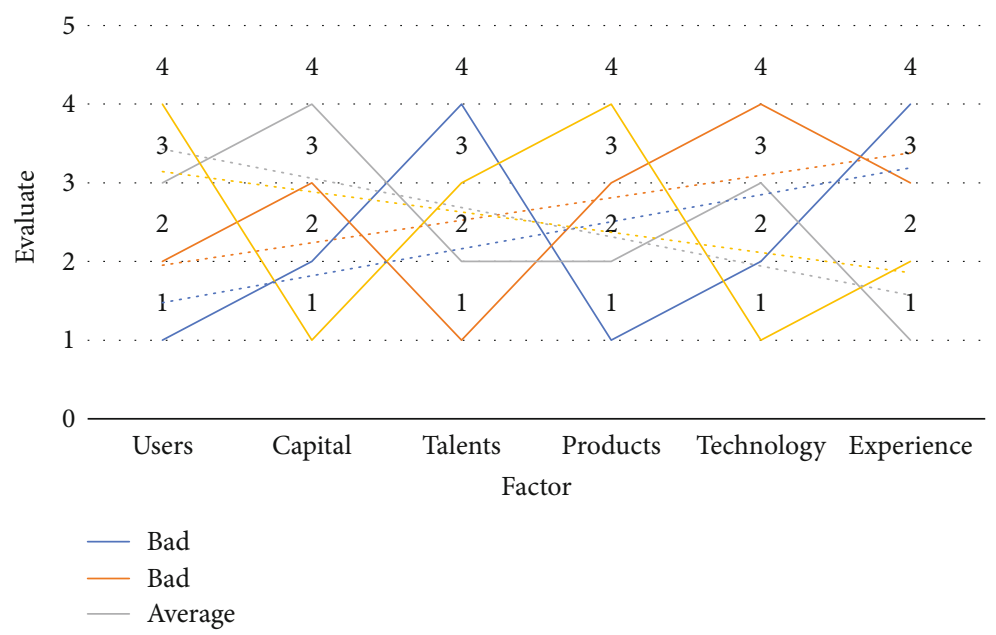

FIgURE 6: Influencing factors and evaluation criteria.

From the perspective of big data globalization strategy innovation, CBEC enterprises need to continuously strengthen bilateral regional cross-border data flow cooperation and need to carry out data fusion and platform docking with government big data platform. One road policy, the state will not only establish a market leading edge in the region but also promote the integration of industrial optimization and rational allocation of resources. The government and CBEC enterprises need to continuously expand the docking degree of the platform, optimize the trade process, and strengthen the supervision. Through the continuous process optimization and industrial upgrading of the bonded zone and free trade zone, CBEC enterprises can make the innovation of decision-making and operation more suitable for the needs of the rapid development of the market and also enable the government to realize dynamic and real-time supervision, to ensure the healthy and orderly development of CBEC industry, as shown in Figure 7.

Cross-border e-commerce enterprises need to have a full understanding of the globalization strategy. By intervening in the big data factors inside and outside the organization, they can make the organization's information communication more rapid and accurate, make the organization's management level flatter, reduce the decision-making level, simplify the operation process, improve the quality of products and services, and increase customer satisfaction, as shown in Figure 8.

From the perspective of big data platform business strategic innovation, CBEC enterprises need to innovate in the form of platform strategic solutions from the perspectives of product, industry, supply chain, and new economic form. Cross-border e-commerce enterprises need to establish and improve $\mathrm{B} 2 \mathrm{~B}$ and $\mathrm{B} 2 \mathrm{C}$ product visualization trading platform, classify, integrate, and analyze business data with visualization big data, and adopt big data precision marketing strategy to realize the promotion of low-cost and accurate products and services. According to the industrial characteristics of CBEC, establish and improve cross-border financial service platform to achieve industrial agglomeration with low-cost funds. 


\section{Top big data applications}

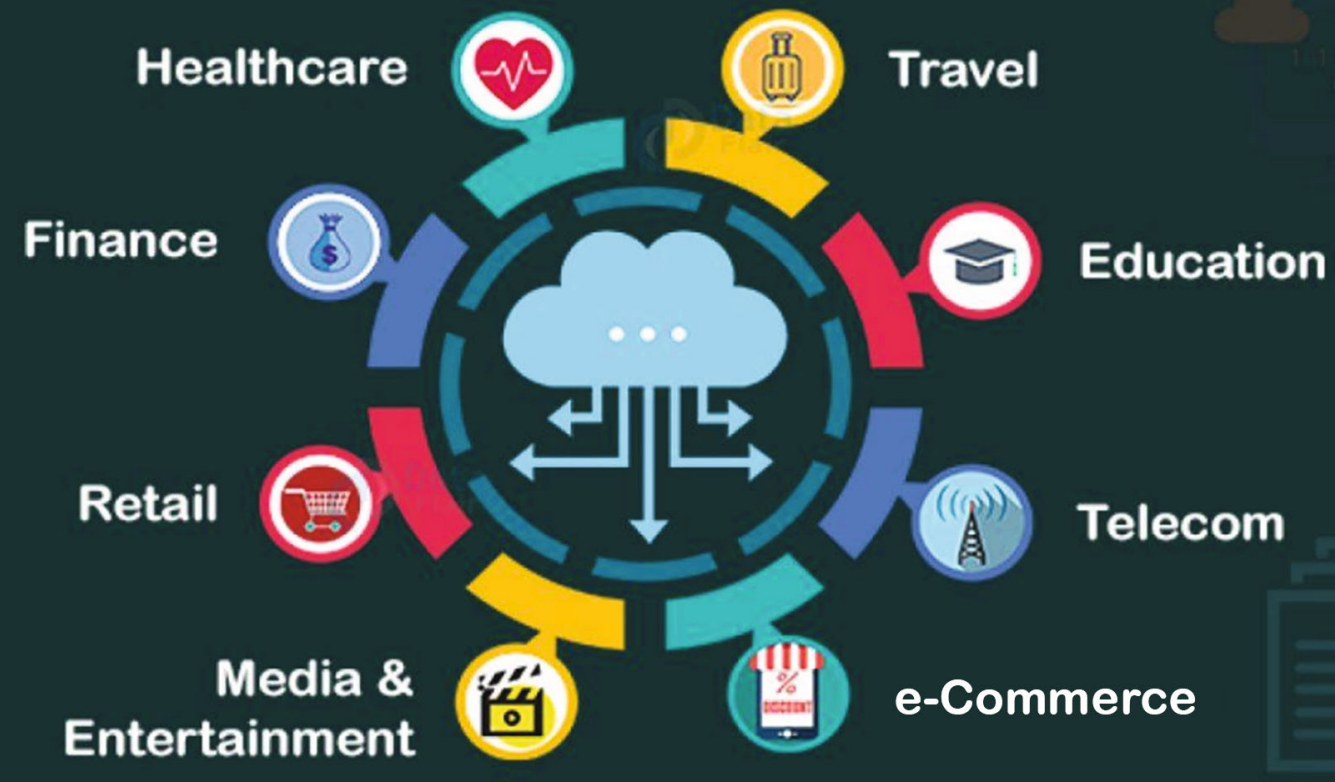

FIGURE 7: Data fusion of enterprise e-commerce platform (http://alturl.com/9b7yp).

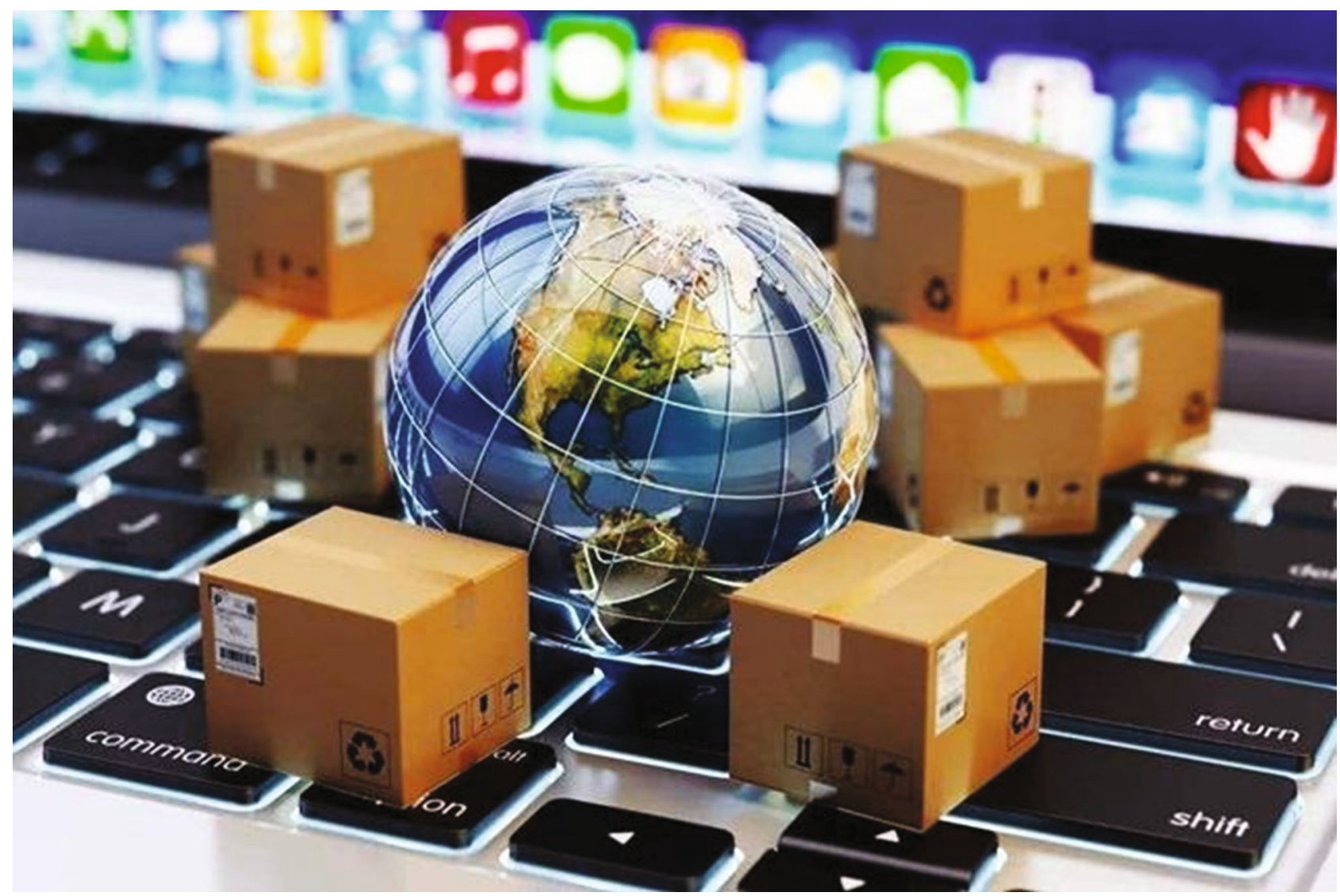

FIgURE 8: Cross-border e-commerce industry development (http://alturl.com/s2fk4). 


\section{Conclusion}

In this paper, the data fusion algorithm method, data fusion network model, quaternion method, big data decision intelligent perception system framework design experiment, and CBEC user experiment are used to study the CBEC big data decision intelligent perception system based on data fusion. With the continuous development of Internet technology and big data application, data-driven and technologydriven business model innovation of modern enterprises emerges endlessly. With the change of social, economic, and related industrial structure, the external environment of enterprises is also changing rapidly. Customer orientation to meet personalized needs and customized services has become the core business focus of enterprises. Based on previous scholars' research, the thinking and theory of big data and enterprise business model innovation are more perfect. Based on these thoughts and theories, modern enterprises find the theoretical basis and thinking direction in the way of big data application and business model innovation. Few scholars have systematically discussed the driving force of business model innovation, and few scholars have conducted in-depth research and discussion on the relationship between business model innovation and the development of enterprise strategy, industry, and national strategy. Previous scholars have done a lot of research on business model innovation, but most of them focus on the process, evaluation, and concept of business model innovation. When they study the process of business model innovation, they tend to put forward the path of business model innovation from one or two aspects; however, few scholars have conducted systematic or strategic discussions on business model innovation in the era of big data.

\section{Data Availability}

No data were used to support this study.

\section{Disclosure}

We confirm that the content of the manuscript has not been published or submitted for publication elsewhere.

\section{Conflicts of Interest}

There are no potential competing interests in our paper.

\section{Authors' Contributions}

All authors have seen the manuscript and approved to submit it to your journal.

\section{Acknowledgments}

This work was supported by Jiangxi Provincial Department of Education Science and technology research project "Jiangxi Province cross border e-commerce industry chain cluster research" (GJJ202505) and Nanchang Institute of Science and Technology introduced talents research start pro- ject "cross border e-commerce industry chain cluster research" (NGRCZX-20-11).

\section{References}

[1] W. Liao, X. Huang, F. Van Coillie et al., "Processing of multiresolution thermal hyperspectral and digital color data: outcome of the 2014 IEEE GRSS data fusion contest," IEEE Journal of Selected Topics in Applied Earth Observations and Remote Sensing, vol. 8, no. 6, pp. 2984-2996, 2017.

[2] K. A. Semmens, M. C. Anderson, W. P. Kustas et al., "Monitoring daily evapotranspiration over two California vineyards using Landsat 8 in a multi-sensor data fusion approach," Remote Sensing of Environment, vol. 185, no. 185, pp. 155170, 2016.

[3] E. Bareinboim and J. Pearl, "Causal inference and the datafusion problem," Proceedings of the National Academy of Sciences of the United States of America, vol. 113, no. 27, pp. 7345-7352, 2016.

[4] M. Berkow, C. M. Monsere, P. Koonce, R. L. Bertini, and M. Wolfe, "Prototype for data fusion using stationary and mobile data: sources for improved arterial performance measurement," Transportation Research Record: Journal of the Transportation Research Board, vol. 2099, no. 1, pp. 102-112, 2018.

[5] M. Schmitt and X. X. Zhu, "Data fusion and remote sensing: an ever-growing relationship," IEEE Geoscience and Remote Sensing Magazine, vol. 4, no. 4, pp. 6-23, 2016.

[6] K. Liu and S. Huang, "Integration of data fusion methodology and degradation modeling process to improve prognostics," IEEE Transactions on Automation Science and Engineering, vol. 13, no. 1, pp. 344-354, 2016.

[7] N. Yokoya, C. Grohnfeldt, and J. Chanussot, "Hyperspectral and multispectral data fusion: a comparative review of the recent literature," IEEE Geoscience and Remote Sensing Magazine, vol. 5, no. 2, pp. 29-56, 2017.

[8] L. Mou, X. Zhu, M. Vakalopoulou et al., "Multitemporal very high resolution from space: outcome of the 2016 IEEE GRSS data fusion contest," IEEE Journal of Selected Topics in Applied Earth Observations and Remote Sensing, vol. 10, no. 8, pp. 3435-3447, 2017.

[9] D. Tuia, G. Moser, B. Le Saux, B. Bechtel, and L. See, “2017 IEEE GRSS data fusion contest: open data for global multimodal land use classification [technical committees]," IEEE Geoscience and Remote Sensing Magazine, vol. 5, no. 1, pp. 70-73, 2017.

[10] B. Pradhan, M. N. Jebur, H. Z. M. Shafri, and M. S. Tehrany, "Data fusion technique using wavelet transform and Taguchi methods for automatic landslide detection from airborne laser scanning data and QuickBird satellite imagery," IEEE Transactions on Geoscience and Remote Sensing, vol. 54, no. 3, pp. 1610-1622, 2016.

[11] F. C. Chen and R. Jahanshahi, "NB-CNN: deep learning-based crack detection using convolutional neural network and Naïve Bayes data fusion," IEEE Transactions on Industrial Electronics, vol. 65, no. 5, pp. 4392-4400, 2018.

[12] F. H. Bijarbooneh, W. Du, E. C.-H. Ngai, X. Fu, and J. Liu, "Cloud-assisted data fusion and sensor selection for internetof-things," IEEE Internet of Things Journal, vol. 3, no. 3, pp. 257-268, 2017.

[13] D. Zhou, K. Zhang, A. Ravey, F. Gao, and A. Miraoui, "On-line estimation of lithium polymer batteries state-of-charge using 
particle filter based data fusion with multi-models approach," IEEE Transactions on Industry Applications, vol. 52, no. 3, pp. 2582-2595, 2016.

[14] Z. Tao and P. Bonnifait, "Sequential data fusion of GNSS pseudoranges and Dopplers with map-based vision systems," IEEE Transactions on Intelligent Vehicles, vol. 1, no. 3, pp. 254-265, 2017.

[15] A. Alofi, A. Alghamdi, R. Alahmadi, N. Aljuaid, and M. Hemalatha, "A review of data fusion techniques," International Journal of Computer Applications, vol. 167, no. 7, pp. 37-41, 2017.

[16] A. Kawa and W. Zdrenka, "Conception of integrator in CBEC," Logforum, vol. 12, no. 121, pp. 63-73, 2016.

[17] Y. H. Hsiao, M. C. Chen, and W. C. Liao, "Logistics service design for cross-border E-commerce using Kansei engineering with text-mining-based online content analysis," Telematics \& Informatics, vol. 34, no. 4, pp. 284-302, 2017.

[18] H. Qian, "Status of cross border e-commerce development against the background of "Silk Road Economic Belt" and measures\%," Journal of Urumqi Vocational University, vol. 25, no. 3, pp. 44-48, 2016.

[19] Y. K. Wang, "Model for evaluating the logistics service quality of cross-border E-commerce enterprises with intuitionistic fuzzy information," Journal of Computational and Theoretical Nanoscience, vol. 14, no. 2, pp. 1136-1139, 2017.

[20] Q. Fan, “An empirical study on cross border e-commerce promoting regional economic I nnovation: taking Taicang City as an example," Jiangsu Science and Technology Information, vol. 36, no. 15, pp. 1-3, 2019.

[21] M. Zhou, Y. Li, M. J. Tahir, X. Geng, Y. Wang, and W. He, "Integrated statistical test of signal distributions and access point contributions for Wi-Fi indoor localization," IEEE Transactions on Vehicular Technology, vol. 70, no. 5, pp. 5057-5070, 2021.

[22] M. Zhou, Y. Wang, Y. Liu, and Z. Tian, “An informationtheoretic view of WLAN localization error bound in GPSdenied environment," IEEE Transactions on Vehicular Technology, vol. 68, no. 4, pp. 4089-4093, 2019. 